\title{
IN UTERO DIAGNOSIS OF DUODENAL ATRESIA
}

\author{
Yadav C $\mathbf{M}^{1}$, Yadav R $\mathbf{P}^{2}$, Krishnanand B $\mathbf{R}^{3}$
}

\section{ABSTRACT}

Duodenal atresia can be fatal unless promptly diagnosed and treated surgically. Death occurs in the newborn secondary to emesis, aspiration, and electrolyte imbalance. Here, we report a case of duodenal atresia diagnosed by ultrasonography at 28 weeks' gestation.

\section{Key Words: Ultrasound, duodenum, atresia.}

\section{INTRODUCTION}

Duodenal atresia or stenosis is the commonest type of fetal small bowel obstruction-its prevalence being one in 10,000 newborns without any association with difference in sex. ${ }^{1,2,3} 40-50$ percent fetuses with duodenal atresia have other associated anomalies. Of these, 30 percent have trisomy 21; 22 percent have malrotation of the gut and 20-30 percent have congenital heart disease .Less commonly associated malformations are renal, hepatobiliary, and tracheo-oesophageal fistula. 45 percent of the cases may be associated with polyhydramnios. ${ }^{2,4,5}$

\section{CASE REPORT}

A 16-year-old primigravida was referred for ultrasonography to determine gestational age, confirm clinically suspected polyhydramnios and rule out any associated congenital malformations.

1. Bir Hospital, Kathmandu, Nepal.

2. Prasuti Griha, Thapathali, Kathmandu, Nepal.

3. Manipal College of Medical Sciences, Pokhara, Nepal.

Address for correspondence : Dr. Chandra Mohan Yadav, M.D.

Radiologist, Bir Hospital, Kathmandu, Nepal.

Ph. No.: 01-522304, Email: drchandramohan@hotmail.com
She was at 28 weeks of gestation. Ultrasound examination revealed intrauterine gestation with single live fetus. It showed polyhydramnios with the amniotic fluid in its deepest pocket measuring $12.0 \mathrm{cms}$. The patient was already in labour. The fetal abdomen showed two fluid-filled spaces (Fig.1) on transverse ultrasound scan, the double-

Fig. 1. Transverse section of fetal abdomen. Asterisks (*) denote stomach, larger anechoic structure and duodenum, smaller one. 'SP' indicates fetal spine.

JNMA, January - March, 2002, 41 
bubble sign associated with duodenal obstruction. Peristalsis was noted in these two fluid-filled structures. The longitudinal scan (Fig.2) showed

Fig. 2

Fig. 2. Longitudinal section of fetal abdomen. Larger sonolucent area, stomach (S), inter-connected with a smaller one, duodenum. 'H' indicates fetal heart.

the larger sonolucent area (stomach) interconnected with another smaller anechoic structure (duodenum). No fluid- filled bowel was observed distal to the duodenum. No other anomalies were demonstrated. The plain X-ray of abdomen (Fig.3)

Fig. 3

Fig. 3. Neonate's X-ray abdomen. Larger radiolucent structure on the left (L) represents stomach and a smaller one on the right, duodenum.

of the neonate done immediately after birth confirmed the in utero diagnosis of complete duodenal obstruction. The proximal left-sided bubble is the stomach while the second bubble represents the proximal duodenum (Fig.3). Laparotomy showed duodenal atresia distal to the ampulla of Vater .The neonate died on the $3^{\text {rd }}$ postoperative day because of severe pneumonia.

\section{DISCUSSION}

Duodenal atresia produces complete proximal bowel obstruction, and, unless an alternative route exists for air to reach the remainder of the small bowel, there is no distal bowel gas. Such an alternative route has been described if the air bypasses the site of atresia via a bifid common bile duct with an insertion both proximal and distal to the atretic segment. The detection of doublebubble sign leads to suspicion of a number of structural anomalies all of which cause duodenal obstruction. There could be various intrinsic (duodenal atresia, duodenal stenosis, and duodenal webs) and extrinsic (annular pancreas, malrotation of the gut with obstruction produced by midgut volvulus or by Ladd bands, and preduodenal portal vein) causes for it. ${ }^{1}$

Most investigators believe that the atresia results from failure of recanalisation of the duodenum, which normally occurs at about 10 menstrual weeks. ${ }^{6}$ Loveday et al first reported prenatal ultrasound detection of duodenal atresia in 1975 . Romero et al reported a case of duodenal atresia diagnosed at 22 weeks' gestation. ${ }^{2}$ This observation conflicts with some reports stating that serial sonography cannot detect duodenal atresia until the third trimester. There are several possible explanations for such occurrence. The skill of the sonographer might be at fault for late detection. Alternately, the fetus might not swallow sufficient amount of fluid to cause dilatation of stomach and duodenum until late in the pregnancy. ${ }^{2}$

Pritchard found the volume of amniotic fluid swallowed by a normal fetus was $7 \mathrm{ml} / 24$ hours at 16 weeks' gestation and $20 \mathrm{ml} / 24$ hours at 28 weeks'. Late in pregnancy, perhaps around 29 weeks' gestation, the 
volume of amniotic fluid swallowed might exceed the resorptive capacity of gut and result in dilatation. ${ }^{2}$

At birth, newborns are generally well hydrated and have adequate liver stores of glycogen. However, they can deteriorate rapidly, and by 72 hours of age, their ability to respond to stress decreases. A prompt diagnosis of duodenal atresia is therefore important to prevent complications related to diagnostic delay, such as dehydration, electrolyte imbalance, or aspiration pneumonia. ${ }^{8}$

Antenatal diagnosis of duodenal atresia is made by sonographic image of the double-bubble sign (Fig.1) on a transverse section of the fetal abdomen. This image results from the dilatation of the stomach and duodenum proximal to the atretic area. A connection between the two "bubbles" must be demonstrated. Otherwise one cystic mass, such as choledochal cyst or omental cyst, might be seen in association with a large stomach and confused with duodenal atresia. ${ }^{9}$

On occasion, a coronal scan of normal stomach can produce an image similar to that that of a duodenal atresia because of the presence of prominent incisura angularis. Transverse scan will demonstrate the depth of the incisura and solve the diagnostic problem. ${ }^{3}$ Once a positive diagnosis is made, a careful search for associated anomalies is important. Because about 30 percent of infants with duodenal atresia have trisomy 21 , the parents should be offered fetal karyotype determination. Recognition of trisomy 21 before the viability offers the parents the option of pregnancy termination. After viability, a diagnosis of trisomy 21 may not alter the obstetric management, but the parents may benefit from knowing a chromosomally abnormal infant in advance. ${ }^{3}$ The risk of recurrence of duodenal atresia in a subsequent pregnancy is 1 per 10,000 and the risk for the offspring of an affected person is the same?

Congenital intrinsic obstruction of the bowel occurs most commonly in the duodenum. If one portion of the bowel is atretic, other segments may be involved. Atresia may affect duodenum proximal ( $20 \%)$ or distal $(80 \%)$ to the ampulla of Vater. ${ }^{10}$

Air, either swallowed or injected into the gastrointestinal tract, is an excellent contrast agent for the delineation of complete proximal intestinal obstruction. ${ }^{11}$ In our case, no fluid-filled distal bowel loop was visualized on ultrasound.This was confirmed by the plain X-ray of the abdomen (Fig.3). A contrast study is indicated, if the plain skiagram of the neonate shows air scattered in the lower abdomen indicative partial duodenal obstruction. Barium enema should be done as an emergency procedure to rule out bowel malrotation.

\section{REFERENCES}

1. J effrey Traubici. Signs in Imaging.Radiology.2001; 220:463-464

2. Nelson LH, Clark CE, Fishburne J I, et al. Value of serial sonography in the in utero detection of duodenal atresia. Obst Gynecol. 1982; 59: 657.

3. Romero J, J eanty P, Pillu G, et al. The prenatal diagnosis of duodenal atresia. Does it make any difference? Obst Gynecol.1988; 71:739-741.

4. Burton DJ, Filly RA. Sonographic diagnosis of amniotic band syndrome. AJ R. 1991; 156:555.

5. Fiedler JM, Phelan JP. The amniotic band syndrome in monozygotic twins.Am J Obst Gynecol.1983; 14:863.

6. Ruth BG. Ultrasound evaluation of fetal abdomen. In: Callen's Ultrasonography in Obstetrics and Gynaecology, $3^{\text {rd }}$ edn: W.B.Saunders Company 1994; 16:347-369.

7. Loveday BJ , Barr J A, Aitken J. The intrauterine demonstration of duodenal atresia by ultrasound. Br J Radiol.1975; 48:1031.

8. Ghory MJ, Sheldon CA. Newborn surgical emergencies of the gastrointestinal tract. Surg Clin North Am. 1985; 65: 1083.

9. Dewbury KC, Auwihare APR, Birch S], et al. Prenatal ultrasound demonstrations of a choledochal cyst. Br J Radiol. 1980; 53: 906.

10. Ajit SA, Tushar W, PradipJJ, Prakash V. Prenatal diagnosis of duodenal atresia by Ultrasound. Indian J Pediatr. 1991; 58: 145-147.

11. Cohen HC, Haller JO, Mestel AC, Coren C,Schechter S, EatonDH. Neonatal duodenum: Fluid-aided US-examination. Radiology 1987; 64:805-809. 